\title{
Failure behaviour of short-fibre-reinforced PBT composites: Effect of strain rate
}

\author{
Dani Abdo $^{\mathrm{a}, \mathrm{b}}$, Andrew Gleadall ${ }^{\mathrm{a}}$, Vadim V. Silberschmidt ${ }^{\mathrm{a}, *}$ \\ ${ }^{a}$ Wolfson School of Mechanical, Electrical and Manufacturing Engineering, Loughborough University, Loughborough, UK \\ ${ }^{\mathrm{b}}$ School of Mechanical Engineering, Rheinische Fachhochschule Koeln, Cologne, Germany
}

\begin{abstract}
A B S T R A C T
Thermoplastic polyester elastomer (TPEE), one of thermoplastic elastomers, suitable for blending with polybutylene terephthalate (PBT), recently attracted significant attention thanks to its beneficial effects on the impact strength of fibre-reinforced PBT composites. Short-fibre-reinforced PBT/TPEE composites are used for critical components in the automotive industry, including electrical connectors. However, there is a lack of knowledge of their dynamic behaviour and microstructural processes underpinning their failure. The aim of this paper is to study the rate-dependent behaviour of such composites at different strain rates, assessing the strain-rate dependency of mechanical properties of short-glass-fibre-reinforced (SGFR) PBT and analyse the microstructural features of its failure at various loading rates. For the first time, standard SGFR PBT and TPEE-blended SGRF PBT were investigated under uniaxial tensile testing at different loading rates ranging between $2 \mathrm{~mm} / \mathrm{min}$ and $400 \mathrm{~mm} / \mathrm{min}$ (corresponding to $0.003 \mathrm{~s}^{-1}$ and $0.6 \mathrm{~s}^{-1}$ strain rates). Fracture surfaces obtained at different loading rates were examined with scanning electron microscopy (SEM) to investigate brittle/ductile properties. The results in dicated that ultimate tensile stress of PBT composites is strain-rate sensitive. Both damage initiation and a rate of crack growth were significantly affected by blending with TPEE and demonstrated dependence on the loading rate. SEM analysis confirmed the effects of blending and strain rate on failure behaviour manifested in varying character fracture-surface morphology and microstructural features of the matrix. Micrographs demonstrated that interfacial matrix-fibre bonding was weakened by the addition of TPEE. Additionally, a strong relationship between the size of ductile areas of fracture surfaces and the loading rate was observed, indicating higher plastic deformation of the matrix for PBT TPEE composite.
\end{abstract}

\author{
Keywords: \\ Automotive failures \\ Fracture surfaces \\ Strain rate \\ Failure analysis
}

\section{Introduction}

To avoid synthesis of new structural materials and related investments in expensive polymerisation equipment, polymer blending became more attractive over the years. Blending of two polymers provides a relatively simple and cost-effective method to obtain new materials with enhanced properties without losing the material's original advantages [1,2].

Short-fibre-reinforced polybutylene terephthalate (PBT) is widely used in the automotive industry, especially in electrical applications, making it one of the most frequently employed polymers in production of electrical connectors and interfaces. Such electrical components are critical and demanded to guarantee required performance. The functioning of active vehicle safety systems,

\footnotetext{
* Corresponding author.

E-mail address: V.Silberschmidt@lboro.ac.uk (V.V. Silberschmidt).
} 
such as anti-lock braking and electronic stability program as well as passive safety systems (airbags, seatbelt tensioners, etc.), depends on reliable design and materials of electrical connectors. These connectors are usually manufactured using a single material type via injection moulding, with connectors' snap fits being a reason of failure or breakage as a result of dynamic interaction between a stationary connector's body and its movable snap fit. One major malfunction cause of electrical connectors is the breakage of snap fits during assembly or vehicle's lifespan, which can result, in turn, in a total or partial system failure. Additionally, in service life, such snap fits are subjected to multiaxial forces and dynamic loads, which produce dynamic states of stress [3]. So, since these components are applied in dynamically loaded critical systems, there is an obvious need to elucidate the dynamic behaviour of SGFR PBT TPEE composites.

PBT, a linear aromatic polyester with high-performance semi-crystalline resin, is one of the most versatile engineering thermoplastics. It has high mechanical strength, excellent processing characteristics and outstanding electrical properties, making it applicable for a broad range of products. To increase the strength, modulus and toughness of the matrix, PBT is usually reinforced with short glass fibres.

Thermoplastic polyester elastomer (TPEE), a new member in the thermoplastic-elastomer (TPE) family, has recently attracted much attention. It is suitable for blending with PBT. Chemically, its hard segments are crystalline polyesters like those of PBT [4], making it compatible with the latter [5]. Recent research was focused on the effect of TPEE on the impact strength of short-glassfibre-reinforced (SGFR) PBT. For instance, Verma et al. [6] observed significant improvement in the impact toughness of PBT/TPE blends, but a decrease in tensile strength, with increasing TPE content. The effect of TPE on impact strength and properties of SGFR PBT and similar polymers is well studied [5-11]. Still, to the best knowledge of the authors, no studies considered strain ratesensitivity of SGFR PBT (TPEE) composites and its effect on their failure behaviour. Several research-groups investigated tension of short-fibre-reinforced (SFR) thermoplastics under dynamic conditions. Fitoussi et al. [12] and Schoßig et al. [13] observed an increase in strength and stiffness with increasing strain rate. Moreover, they investigated the effect of fibre orientation caused by mould flow on the tensile properties and suggested that, when a strain rate increased, deformation zones around glass fibres acted as a dissipation area, leading to delay in damage initiation. Thanks to its enhanced impact strength, SGFR PBT TPEE was adapted for production of electrical connectors and other critical components, strengthening the demand to study the mechanical behaviour of this composite and analyse its failure mechanisms.

This study investigates the rate-dependent tensile behaviour and failure mechanisms of SGFR PBT composites for the first time, combining quasi-static and dynamic tensile tests. The complexity of failure behaviour of SGFR composites is greatly affected by its constituents; matrix and reinforcement. The matrix properties and heterogeneity caused by the distribution of glass fibres are major features of investigation of failure mechanism of these composites. Thomson [14] showed that performance of SGFR plastics depended on stress-transfer capability of fibre-matrix interfaces and that a length and a diameter of fibres did not have a significant effect on the composite's complex modulus. Stress-concentration-induced damage mechanisms were studied by Horst and Spoormaker [15] and Sato et al. [16,17] who found that performance of a composite depended on the properties of fibre-matrix interface and the character of load transmission between these two components. Furthermore, it was concluded that the load-transmission capacity depended on adhesion between glass fibres and a matrix.

In this study, specimens of PBT-GF10 (10\% glass-fibres) and PBT-GF10 blended with 10\% TPEE are tested to failure in tension at different loading rates for the first time. The effect of TPEE on SGFR PBT along with the loading-rate-dependency of both materials is evaluated in terms of Young's modulus, tensile strength, strain at ultimate stress and elongation at break. SEM micrographs of resultant morphology of fracture surfaces are analysed for a range of loading rates, to elucidate the effect of loading rate and TPEE on the morphological characteristics of the materials and characterise its failure mechanism. This is achieved by studying the ductile/ brittle behaviour observed on fracture surfaces and evaluating the effect of TPEE and strain rate on the interfacial fibre-matrix bonding.

\section{Experimental setup and methodology}

\subsection{Materials and specimens}

In this paper, two PBT composites are investigated. The first is standard polybutylene terephthalate with $10 \mathrm{wt} \%$ glass-fibre content referred to as PBT-GF10. PBT-GF10 is used as a reference material to evaluate the effect of TPEE on SGR PBT composites.

The second material, polybutylene terephthalate with $10 \mathrm{wt} \%$ glass fibres and $10 \mathrm{vol} \%$ thermoplastic polyester elastomer (TPEE),
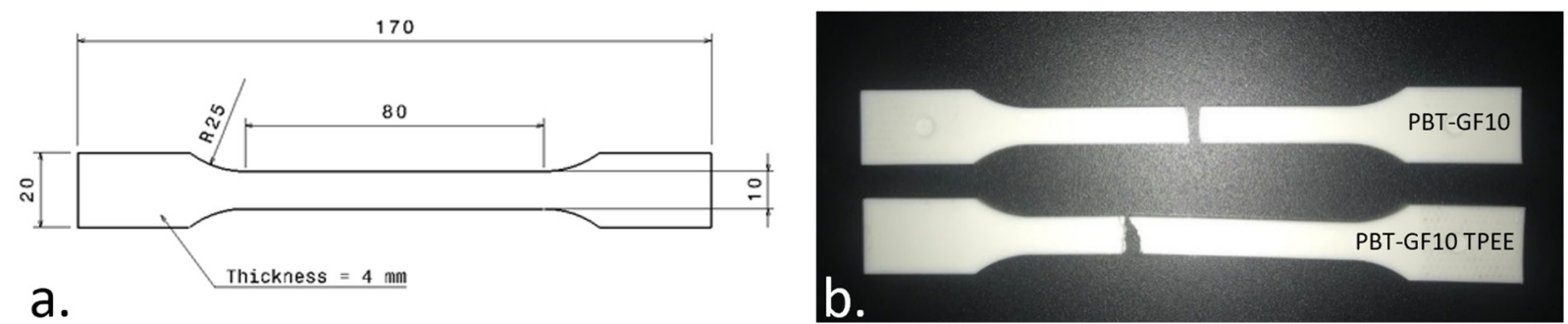

Fig. 1. (a) ISO 527 dogbone specimen (all dimensions in mm); (b) Specimens of PBT composites showing no necking after fracture. 


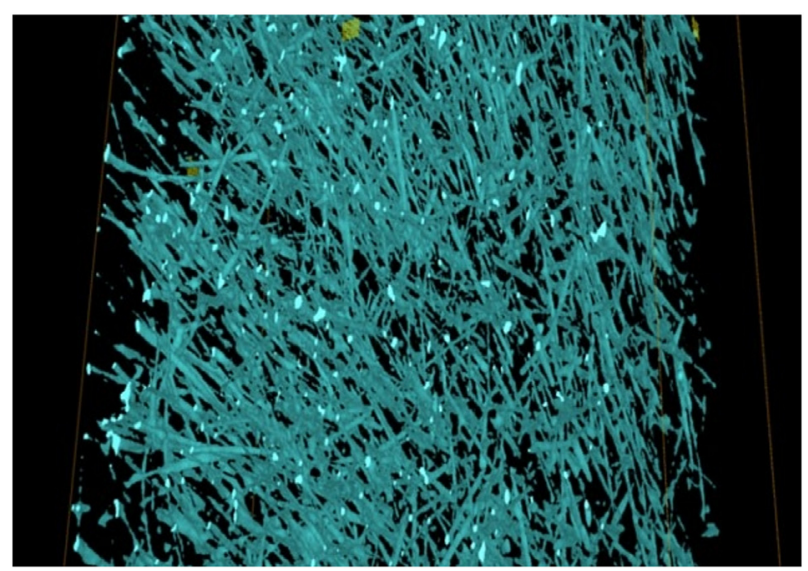

Fig. 2. X-ray CT scan of PBT-GF10 showing fibres orientation in matrix.

is referred to as PBT-GF10 TPEE.

All samples used in experiments were standard ISO 527 dogbone specimens (see Fig. 1a) produced via injection moulding. X-ray computer tomography scans showed that fibres were distributed randomly in the matrix, mainly oriented along the flow direction in the mould, which was in the loading direction of the specimens for tensile tests (Fig. 2).

\subsection{Mechanical characterisation}

Specimens were conditioned at 50\% relative humidity at room temperature for 4 weeks. Tensile tests were performed on a Zwick Z010 machine equipped with a $10 \mathrm{kN}$ load cell. All tests were performed using a contact type extensometer with a $10 \mathrm{~mm}$ gauge length. Specimens of both materials were tested up to failure at four different loading rates. Crosshead travel speeds were of 2, 20, 200 and $400 \mathrm{~mm} / \mathrm{min}$ were chosen on a logarithmic scale according to the values recommended in ISO 527 standard [18]. The last magnitude was employed instead of the recommended value of $2000 \mathrm{~mm} / \mathrm{min}$ to match a potential usability envelope of the studied materials. It should be noted here that the term quasi-static is used for a loading rate $2 \mathrm{~mm} / \mathrm{min}$ referring to a low crosshead speed and the term dynamic used in this paper explicitly refers to crosshead speeds of 20, 200 and $400 \mathrm{~mm} / \mathrm{min}$, representing a higher range of loading rates.

\subsection{SEM observations and morphological characterisation}

Morphological analysis was completed using a Phenom XL scanning electron microscope with a range of accelerating voltage from 5-15 kV. To prevent specimens from charging, a charge-reduction mode, which is presented as low-vacuum mode, was activated. This mode produced best results in terms of noise reduction and quality of micrographs. An additional SEM system (JSM-7500F) with the same range of accelerating voltage was also used. For investigations with this microscope, specimens were sputter-coated with a 10-15 nm thick layer of gold-palladium to provide an efficient charge transfer. The aim of employing two different microscopes and methods was to ensure that there were no losses in image quality due to the charging problem. Gold-palladium coating was effective to obtain high-quality images of the matrix but had a negative effect on observation of matrix residue on pulled-out fibres. All fractographs were studied using the image processing software Image $J$ to analyse and calculate the area of ductile regions on fracture surfaces.

\section{Results and discussion}

To understand the failure mechanism of any composite material, it is essential to know the behaviour and properties of its constituents that affect the overall behaviour of the composite. Multiple studies showed [15-17,19-21] that glass fibres are rate insensitive. Based on this fact, the rate-dependent failure behaviour of PBT composites depends mainly on two factors: a character of interfacial bonding between the matrix and glass fibres and rate sensitivity of the matrix.

\subsection{Loading-rate-dependent tensile behaviour of PBT-GF10 and PBT-GF10 TPEE}

The tensile measurements of PBT-GF10 and PBT-GF10 TPEE were performed at different loading rates, 2, 20, 200 and 400 mm/ min and the obtained stress-strain curves are shown in Fig. 3 (a) and (b), respectively. No visual necking or yielding phenomenon was noticed during the tensile tests for both PBT-GF10 and PBT-GF10 TPEE specimens as obvious from Fig. 1(b). The ultimate tensile strength of both PBT composites could be deduced from the quasi-static tensile measurements performed at $2 \mathrm{~mm} / \mathrm{min}$, whereas the values of ultimate tensile strength measured during the dynamic conditions at loading rates 20,200 and $400 \mathrm{~mm} / \mathrm{min}$ correspond to 

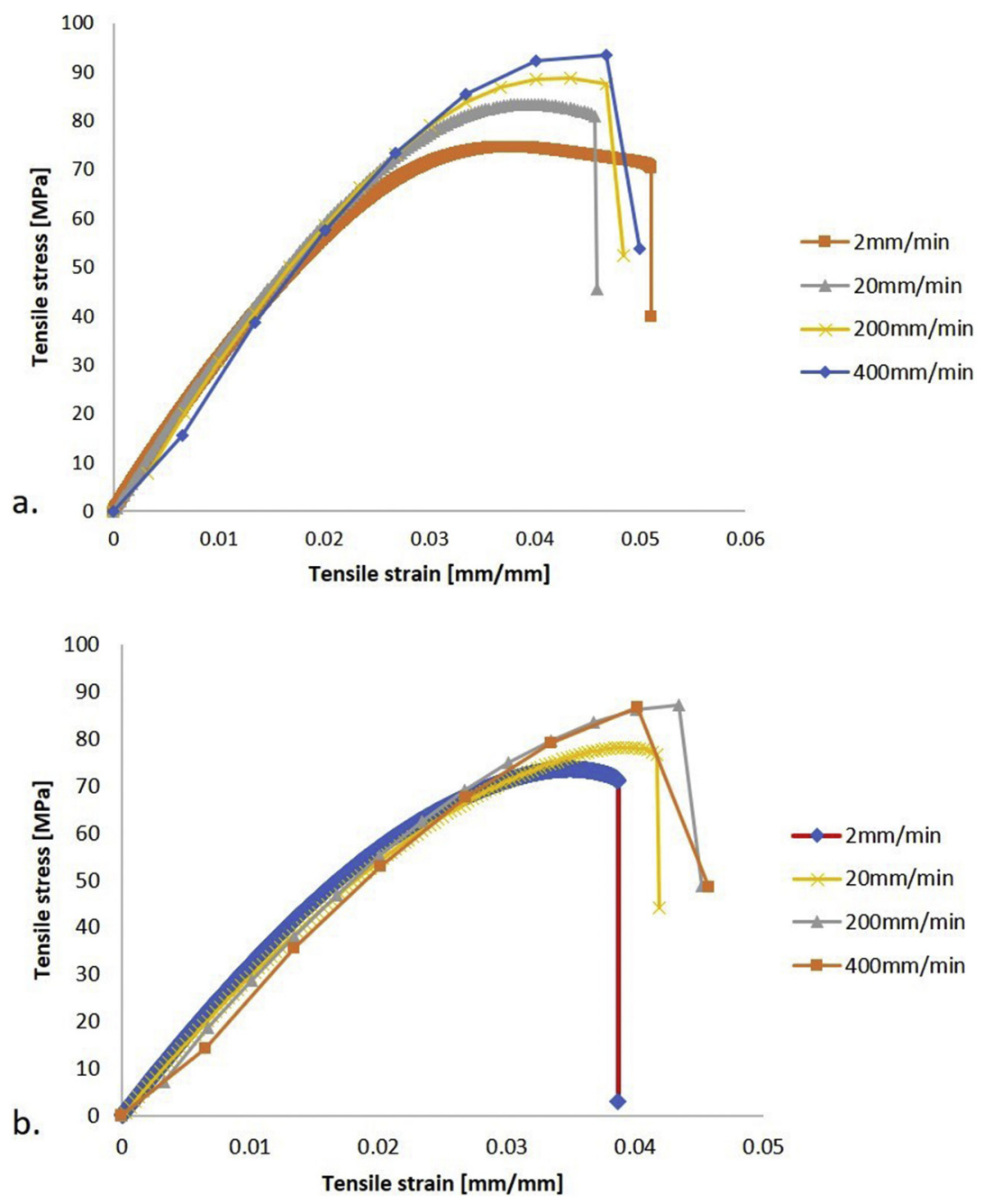

Fig. 3. Experimental stress-strain curves of PBT composites at various loading rates: (a) PBT-GF10; (b) PBT-GF10 TPEE.

dynamic responses: the highest point of these curves indicates the dynamic tensile strength [22]. The variation in tensile responses demonstrates sensitivity of both materials to loading rates, with values of the Young's modulus, UTS and strain at break, which are discussed in the following sections under quasi-static loading and dynamic loading conditions, varied with increasing loading rate.

\subsection{Behaviour under quasi-static and dynamic conditions}

As can be seen from quasi-static tensile responses, the stress-strain curves are relatively linear in the initial stages, followed by a non-elastic regime with gradual strain hardening. This behaviour is related to initiation of plastic deformation and damage. The latter might occur for the following reasons: failure of the matrix, failure of glass fibres, or interfacial debonding between glass fibres and the matrix. Fibre failures should be relatively rare since their tensile strength can reach $2500 \mathrm{MPa}$ [23], whereas the maximum macroscopic stress in this study was $<100 \mathrm{MPa}$.

After the strain-hardening stage, both curves reached UTS before starting to decline gradually to full failure. Apparently, the decrease in tensile-stress value after UTS extended to higher strains for PBT-GF10 than PBT-GF10 TPEE. The rate of crack propagation, which led to final fracture, is related to the difference between the strain at UTS and the strain at break. This rate, which had a direct effect on a premature fracture of the specimen, was significantly higher for PBT-GF10 TPEE: elongation at break at $2 \mathrm{~mm} / \mathrm{min}$ was 5\% for PBT-GF10 and 3.8\% for PBT-GF10 TPEE. This is an indication of more active damage initiation in the microstructure of PBT TPEE composite.

The stress-strain curves of responses of PBT-GF10 composites to dynamic loading conditions (20, 200, 400 mm/min) are similar in character to those under quasi-static loading. Apart from a higher nonlinearity of their initial and final stages, they demonstrate the same features of strain hardening towards UTS followed by a gradual stress decline. However, the extent of post-UTS stress reduction for loading rates 200 and $400 \mathrm{~mm} / \mathrm{min}$ is smaller. Interfacial debonding between the matrix and glass fibres is normally the main 

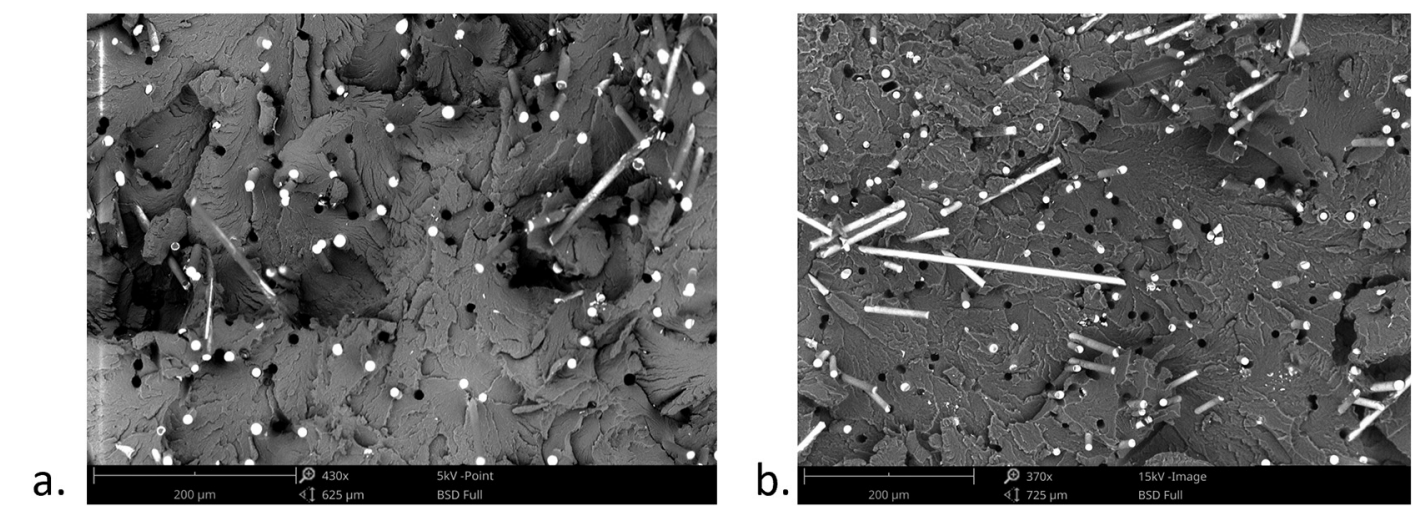

C.
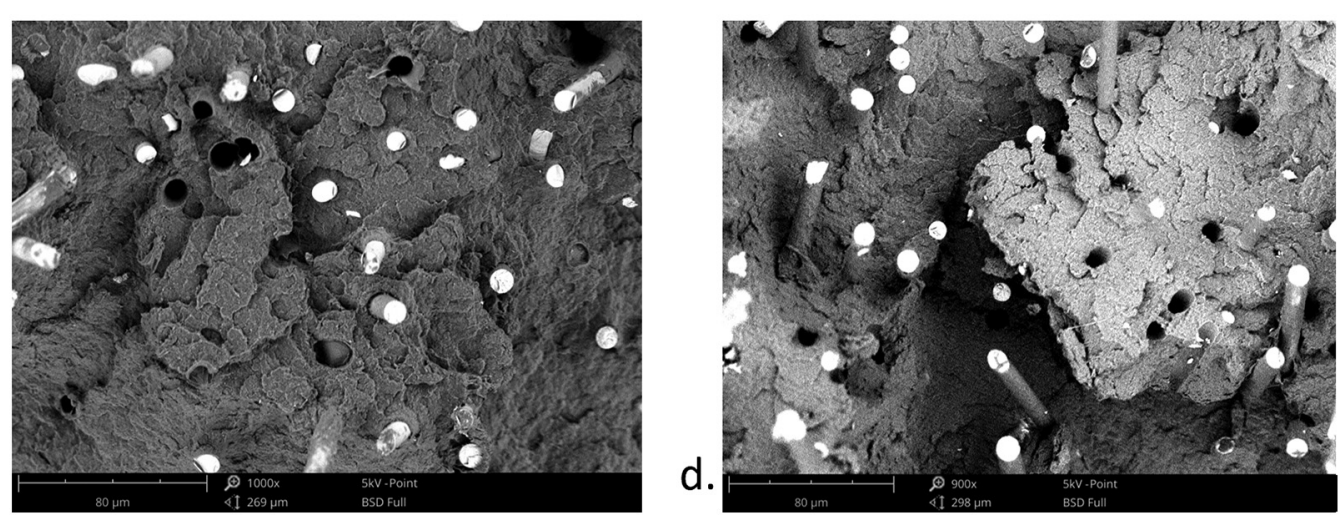

Fig. 4. Morphology of tensile fracture surface of PBT composites; (a) PBT-GF10/quasi-static loading; (b) PBT-GF10/dynamic loading; (c) PBT-GF10 TPEE/quasi-static loading; (d) PBT-GF10 TPEE/dynamic loading.

cause of failure prior to final fracture, which is clearly noticeable in the fractographs analysed in this study. The effect of loading rate observed from the stress-strain curves could be mainly related to the rate-sensitivity of the PBT matrix and to a rate-dependent behaviour of the interfacial matrix-fibre adhesion. To get better understanding of the microstructural contribution, morphology of fracture surfaces was analysed with SEM (Fig. 4).

Generally, rougher and more textured surfaces were caused by dynamic conditions than those generated under quasi-static conditions, as seen in the fracture surfaces in Fig. 4. Several studies of different composite materials produced similar observations. This phenomenon is related to increased fracture toughness of a matrix under dynamic loading [24].

The obtained experimental results clearly demonstrate the effect of strain rate on the Young's modulus for the studied range of loading rates; the qualitative analysis is shown in Fig. 5. The values of the Young's modulus in quasi-static loading were $4200 \mathrm{MPa}$ for

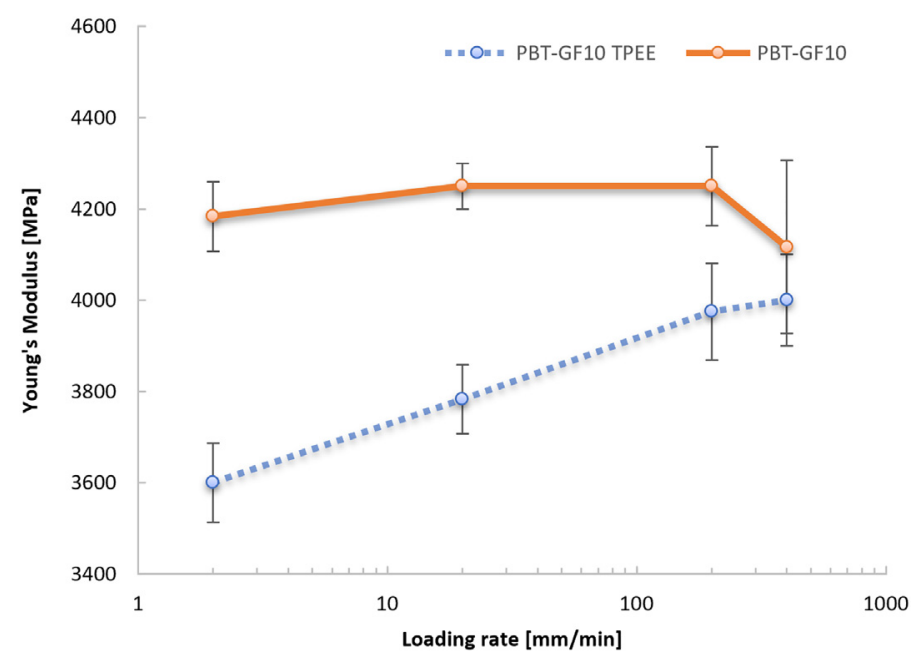

Fig. 5. Variation of Young's modulus for PBT-GF10 and PBT-GF10 TPEE with loading rate. 


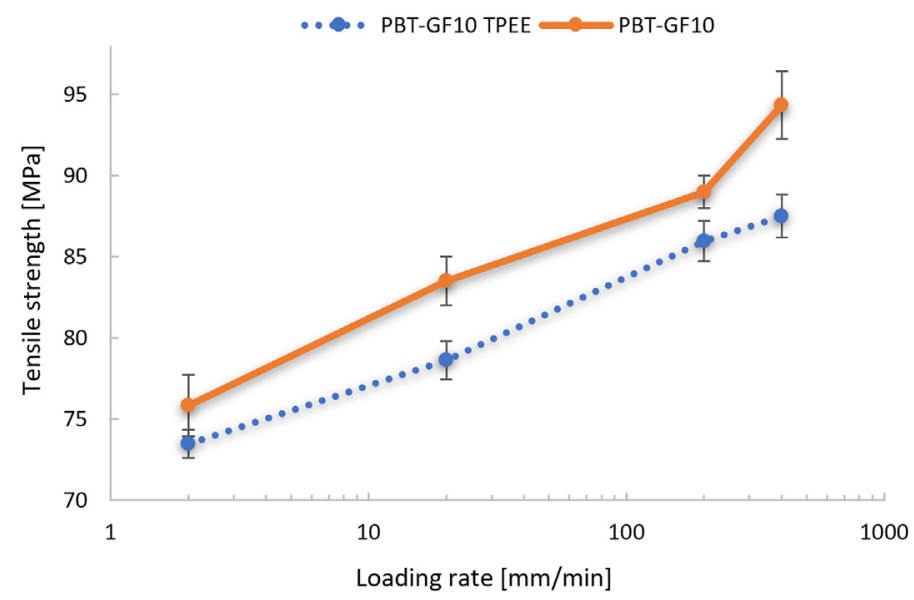

Fig. 6. Effect of loading rate on tensile strength of PBT-GF10 and PBT-GF10 TPEE.

SFGR PBT-GF10 and 3600 MPa for PBT-GF10 TPEE, showing a negative effect of TPEE on the level of stiffness in the latter. Under dynamic conditions, the value of Young's modulus for PBT-GF10 was not greatly affected by the increasing loading rate, staying effectively constant (considering the spread of the experimental data).

On the other hand, the material blended with TPEE demonstrated a clear trend of increasing Young's modulus with the growing loading rate - from $3600 \mathrm{MPa}$ at $2 \mathrm{~mm} / \mathrm{min}$ to $4000 \mathrm{MPa}$ at $200 \mathrm{~mm} / \mathrm{min}$. Importantly, the difference between the values of the Young's modulus diminished consistently, nearly vanishing at $400 \mathrm{~mm} / \mathrm{min}$ (some $3 \%$ for the average values).

Tensile strength of PBT-GF10 and PBT-GF10 TPEE composites is plotted as function of loading rate in Fig. 6. Apparently, the magnitude of tensile strength for both composites strongly depends on the rate of the applied load: the overall increase in ultimate tensile strength for PBT-GF10 and PBT-GF10 TPEE in the range between $2 \mathrm{~mm} / \mathrm{min}$ and $400 \mathrm{~mm} / \mathrm{min}$ was $24 \%$ and $14 \%$, respectively. Both materials obviously demonstrated strain-rate-induced strengthening. At loading rate of $2 \mathrm{~mm} / \mathrm{min}$, PBT-GF10 had a tensile strength of $76 \mathrm{MPa}$. It increased by $10 \%$ at loading rate $20 \mathrm{~mm} / \mathrm{min}$ reaching a value of $83.5 \mathrm{MPa}$, followed by subsequent increases to $89 \mathrm{MPa}$ and $94 \mathrm{MPa}$ at $200 \mathrm{~mm} / \mathrm{min}$ and $400 \mathrm{~mm} / \mathrm{min}$, respectively. The respective values for PBT-GF10 TPEE were $73 \mathrm{MPa}$ (at $2 \mathrm{~mm} / \mathrm{min}$ ), $78 \mathrm{MPa}, 85.9 \mathrm{MPa}$ and $87.5 \mathrm{MPa}$ (at $400 \mathrm{~mm} / \mathrm{min}$ ). Although both materials exhibited similar increasing trends, the graphs also show the effect of TPEE: the tensile-strength values of PBT-GF10 were consistently higher than those of PBTGF10 TPEE.

The loading rate also affected deformational characteristics of the studied composites. Its effect on strain at UTS is shown in Fig. 7 (a). This strain represents the plastic deformation capacity of a material. The effect of TPEE on this parameter was clearly observed under quasi-static conditions, manifested by a 10\% drop in its magnitude. In dynamic loading regime, as observed in the graphs, the value of strain at UTS increased for both materials with the increasing loading rate, proving that this value as well was rate-sensitive. In the range of loading rates up to $200 \mathrm{~mm} / \mathrm{min}$, the rate of increase in PBT-GF10 TPEE was greater, showing that its deformation capacity exceeded that of PBT-GF10. Generally, damage initiation and plastic deformation in polymers is a relatively slow process.
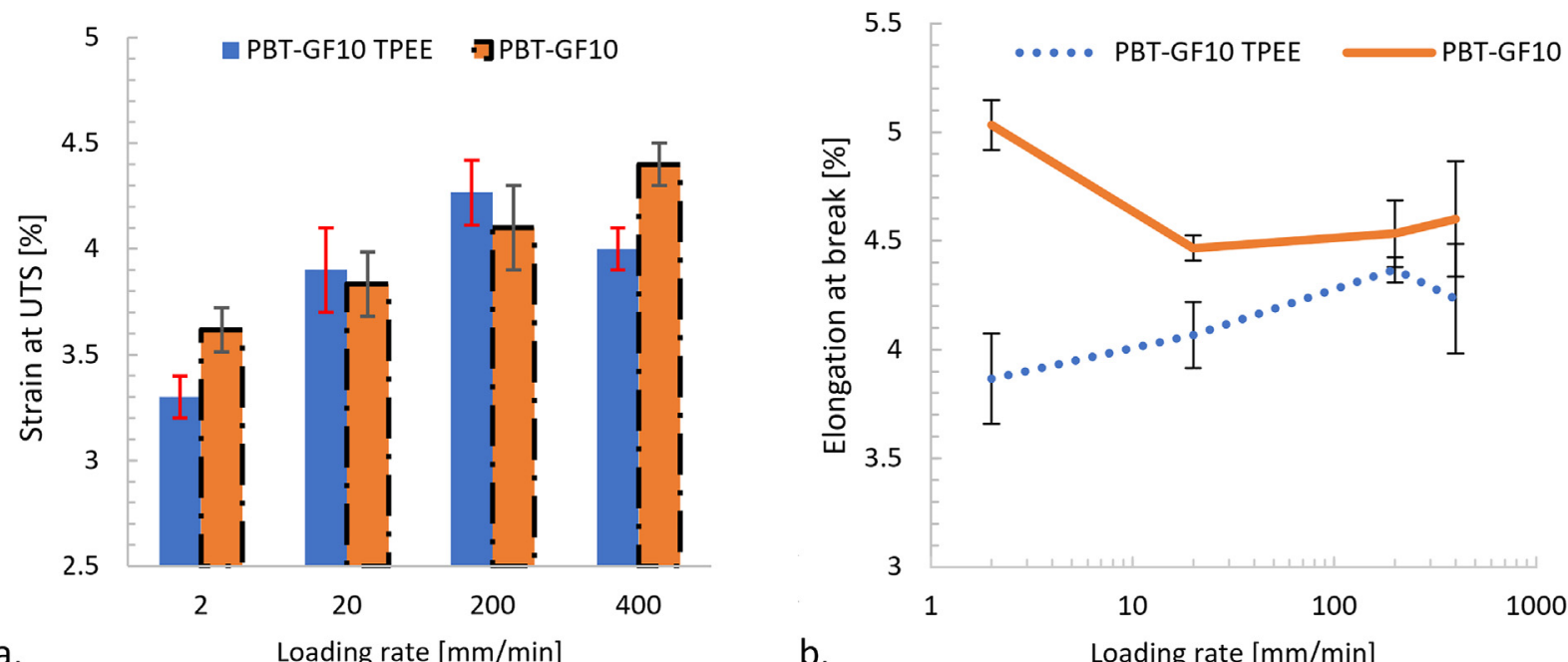

Fig. 7. Effect of loading rates on strain at ultimate stress (a) and elongation at break (b) for PBT composites. 
Due to their viscoelastoplastic behaviour, irreversible deformation in such composites is time-dependent. With increasing loading rates, the materials response to external load becomes restricted. This was observed by several researchers; for instance, Fitoussi et al. [12] stated in their study about the effect of matrix on SFR EPC (ethylene-propylene copolymer) that microcracks were initiated in a ductile manner and needed time to form.

With increasing cross-head velocity, there was less time for damage generation and accumulation, leading to growing levels of strain at UTS in dynamic loading. This was also confirmed by higher microstructural damage observed in PBT-GF10 TPEE as compared to that in PBT-GF10 in quasi-static loading.

The effect of added TPEE on the strain at break was highest under quasi-static conditions, as can be seen in Fig. 7 (b): it diminished by $>20 \%$. Evolution of this parameter under dynamic loading was different in two studied composites: for PBT-GF10, with increasing loading rate, a drop from $5 \%$ to $4.5 \%$ at $20 \mathrm{~mm} / \mathrm{min}$ was followed by a nearly constant level. The results for composite PBT-GF10 TPEE showed a different trend Its value increased at $20 \mathrm{~mm} / \mathrm{min}$ and $200 \mathrm{~mm} / \mathrm{min}$ (to $4.1 \%$ and $4.4 \%$, respectively), decreasing at $400 \mathrm{~mm} / \mathrm{min}$ (a wide spread of experimental data was observed at this rate). So, a transition from quasistatic to dynamic loading of PBT-GF10 TPEE resulted in an increased strain at failure, in contrast to PBT-GF10. As discussed, since glass fibres are rate-insensitive, the observed rate sensitivity in failure strain of both materials is a result of interaction between glassfibres and the matrix and the rate-sensitivity of the latter. This is another indication of the effect of loading rate on the damage evolution in the TPEE composite. This observation confirms that the material is able to withstand higher loads in dynamic loading than in quasi-static one. The increase in failure strain at higher loading rates apparently leads to an increase in fracture toughness, supporting the results of other studies about the positive effect of TPEE on impact strength of PBT composites.

\section{Analysis of fracture surfaces of PBT composites}

\subsection{Brittle and ductile morphological features}

SEM analysis was performed to investigate fracture surfaces of specimens of both studied composites tested in tension at loading rates of 2, 20, 200 and $400 \mathrm{~mm} / \mathrm{min}$.

Typical SEM micrographs of PBT-GF10 and PBT-GF10 TPEE composites presented in Fig. 8 demonstrate major morphological characteristics of their fracture surfaces. Several zones were indicated on the fracture surfaces: Zone A is characterized as having a rough structure, with stretched ligaments of the matrix - a clear indication of its ductile failure mode. Such a structure can be a result of formation of micro cracks at fibre-matrix interface and their coalescence. The borders of ductile areas are highlighted, enclosed with a red line in the fractographs in Fig. 8. The analysis of multiple micrographs for different loading rates demonstrated that there was always only one region of ductile area on each fracture surface. Referring to the fact that the ductile area is the region of crack initiation and stable growth [25], a conclusion can be drawn that for both materials the damage initiation happened at a single region in the matrix. Furthermore, it was noticed that location of ductile area on the fracture surfaces was unpredictable, at all loading rates: ductile areas were found at the edge of a fracture surface for some specimens and randomly in the middle for other specimens. This variability may be due to the inhomogeneity of the composite and the effect of randomly distributed fibres, which cause local areas with low fibre density, as observed in X-ray CT scans of both materials.

The rest of fracture surfaces of the tested specimens demonstrated considerable fibre pull-outs but a relatively smooth surface pattern of the matrix, as seen in Fig. 8, zone B. This is a clear manifestation of a brittle failure mode of the matrix. The brittle area of PBT-GF10 showed a smoother and more planar texture than the brittle zone observed for PBT-GF10 TPEE, which was similar to that reported by Shaaf et al. [26] for unstable crack propagation in PBT-GF30. This zone of PBT-GF10 TPEE had indications of micro-
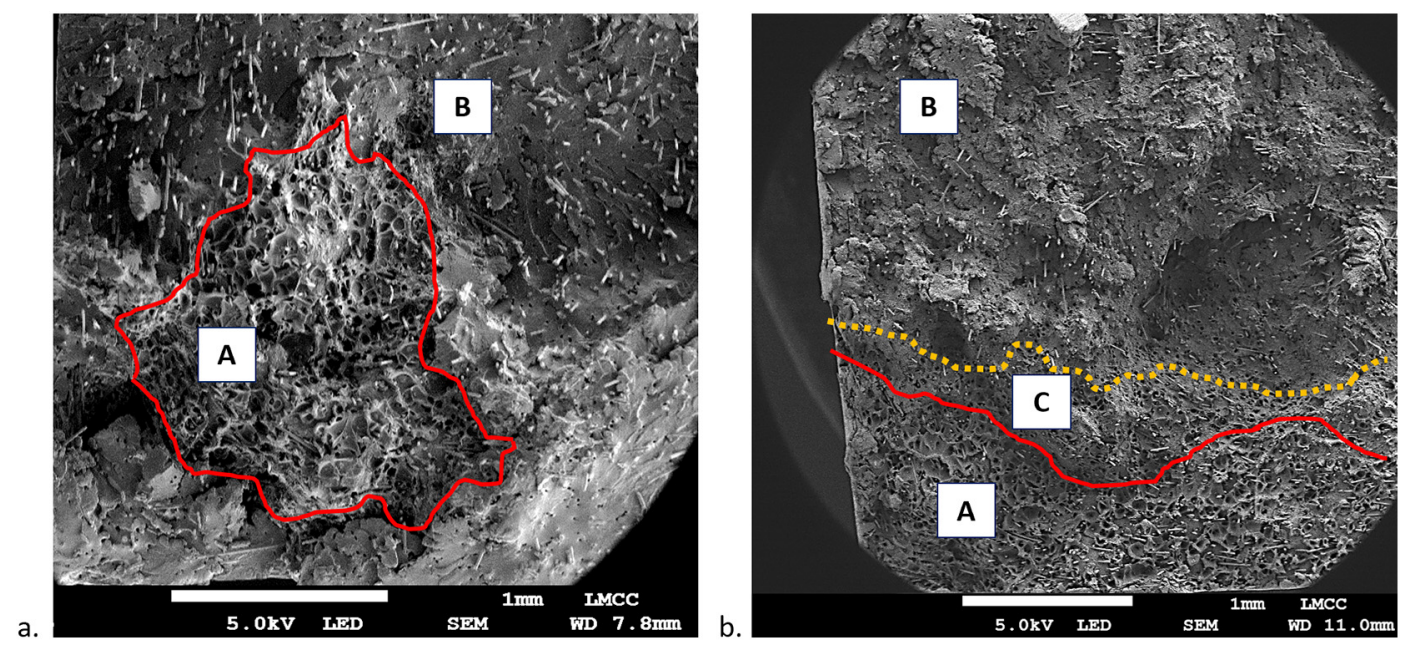

Fig. 8. SEM micrographs showing major types of fracture surfaces - brittle, ductile and transition zones: (a) PBT-GF10; (b) PBT-GF10 TPEE. 

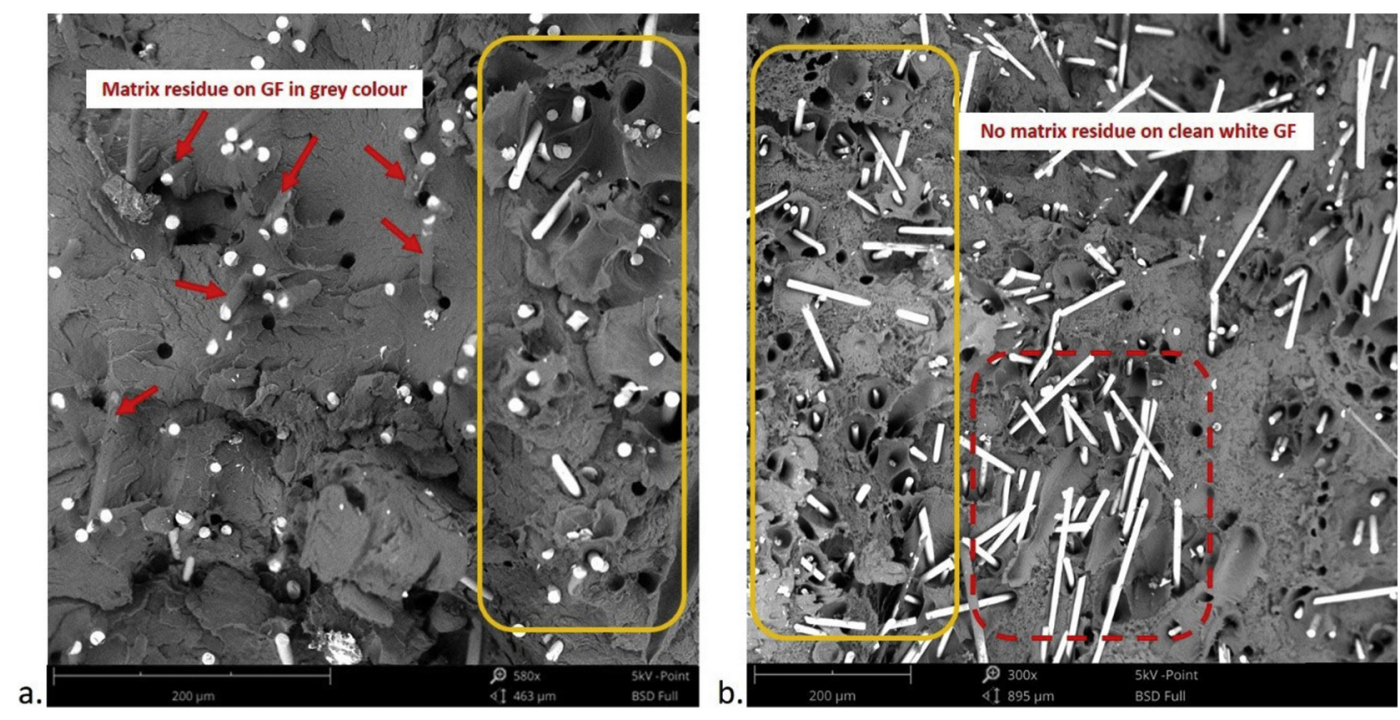

Fig. 9. Micrographs showing layers of matrix on glass fibres: (a) PBT-GF10; (b) PBT-GF10 TPEE.

ductile behaviour responsible for significant surface roughness, not observed for PBT-GF10.

In addition to zones $\mathrm{A}$ and $\mathrm{B}$, zone $\mathrm{C}$ indicated a transition behaviour between the ductile and brittle regimes in PBT-GF10 TPEE. Transition zones are a commonly known morphological feature [27]. It was observed that their size decreased at higher loading rates. No significant transition areas were noticed on fracture surfaces of composite PBT-GF10.

\subsection{Fibre pull-out and fibre-matrix bonding}

The tensile tests performed in this study showed that performance of PBT composites was affected by the addition of TPEE as well as strain rate. Another factor, affecting mechanical properties of glass-fibre reinforced composites, is interfacial adhesion between the matrix and fibres. The difference in interfacial adhesion can be observed in Fig. 9, which shows a significantly greater layer of matrix on pulled-out fibres in PBT-GF10 than PBT-GF10 TPEE. This could explain the higher tensile strength of PBT-GF10 composite. Red arrows in Fig. 9(a) point to fibres covered with relatively thick layer of matrix; this was observed mainly in the brittle zones of the fracture surfaces. Yellow rectangles in Fig. 9 mark parts of the ductile areas, with fibres having no layer of matrix in the ductile region. The slow process of damage initiation in the matrix could be the reason for this phenomenon, allowing a clean matrix-fibre debonding at the interfaces in ductile areas. On the other hand, in PBT-GF10 TPEE, the matrix layer on fibres was thin. Most of the fibres had no traces of matrix on their surfaces in the micrographs, even in regions of brittle failure as seen in Fig. 9(b) marked with a red dashed rectangle, indicating a week fibre-matrix bonding.

The higher tensile strength and stiffness observed for PBT-GF10 was a direct result of significant interfacial bonding observed in its fractographs. Void formation and expansion of cavities formed by pulled-out fibres were observed in ductile and brittle areas for PBT-GF10 TPEE (Fig. 10). The voids formed by fibre pull-outs were more deformed than those in brittle areas. In this composite the deformation was significant under both quasi-static and dynamic conditions (Fig. 10 (c, d)). The cavities formed by fibre pull-outs were stretched at low loading rate as shown in Fig. 10 (c: arrows 1,4 and 5) and dynamic loading Fig. 10 (d). This phenomenon was not observed on fracture surfaces of PBT-GF10, where the voids formed around pulled-out fibres demonstrated no increase in area or shape in both quasi-static and dynamic conditions Fig. 10(a: arrows 1,3 and 5 and b: arrows 1, 2 and 3). This confirms the suggestion that the matrix in the PBT-TPEE composite possesses a higher deformation capability in quasi-static and dynamic loading regimes.

As a conclusion, clear differences in interfacial bonding were found for the two studied materials. The failure initiation mainly occurred in the matrix and, particularly, in a close vicinity to the fibre-matrix interface, resulting in fibre pull-outs; there was no evidence of significant fibre failures. A number of broken fibres (Fig. 10(a: arrow 5; b: arrow 1; c: arrows 1,2,4 and 5) were found on the surfaces, without clear indications whether these fibres were broken during injection moulding, tensile testing or after the experiment. Still, the number of these fibres is not comparable with that of pulled-out ones. Thus, interfacial debonding followed by matrix failure was the dominant mechanism in both cases although demonstrating some differences in the interfacial matrix-fibre adhesion.

\subsection{Effect of loading rate on size of ductile areas}

Analysis of micrographs demonstrated changes in the size of the ductile area with increasing loading rate. To assess this phenomenon quantitatively, the fractographs were analysed using the software ImageJ. Specimens were studied for each loading rate, and the fraction of the fracture surface occupied by the ductile zone, was calculated. The presence of transition areas between the 

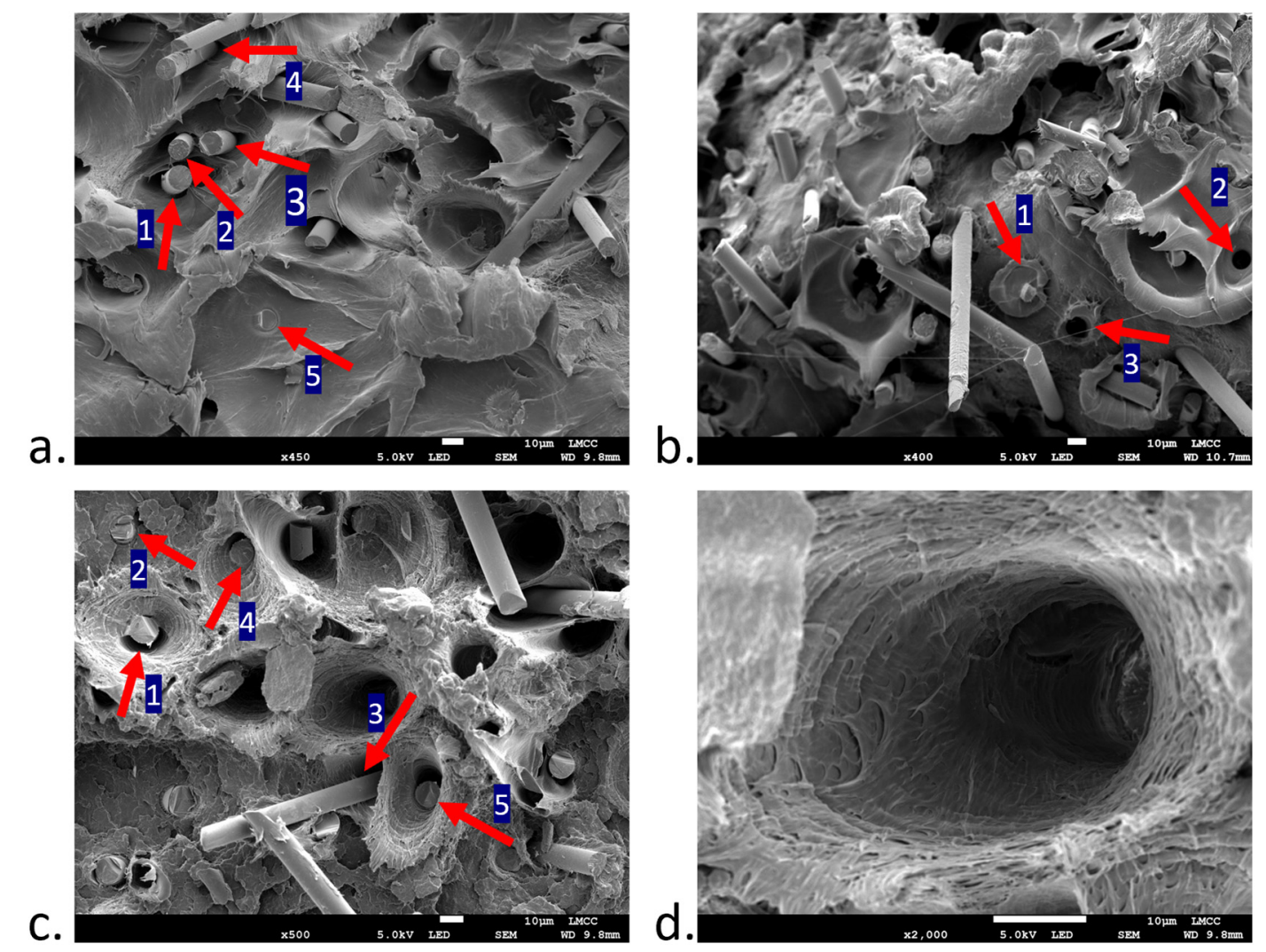

Fig. 10. Void formed by fibre pull-outs under quasi-static and dynamic conditions; (a) PBT-GF10 at $2 \mathrm{~mm} / \mathrm{min}$; (b) PBT-GF10 at $200 \mathrm{~mm} / \mathrm{min}$; (c) PBT-GF10 TPEE at $2 \mathrm{~mm} / \mathrm{min}$; (d) PBT-GF10 TPEE at $200 \mathrm{~mm} / \mathrm{min}$.

ductile and brittle zones on the fracture surface introduced a small error into calculation for ductile areas, which was assessed to be below 3\%, and, hence, did not affect the trends identified in this study. The analysis revealed a strong relationship between the size of ductile areas and the applied loading rate. It was observed that the fraction of these ductile zones on fracture surfaces reached maximum under quasi-static loading conditions. This fraction decreased with increasing loading rates in both materials (Fig. 11).

However, the ductile areas in PBT-GF10 TPEE were greater than those of PBT-GF10 at all loading rates. At $2 \mathrm{~mm} / \mathrm{min}$, the ductile area for PBT-GF10 TPEE was nearly two times larger than that in PBT-GF10. This difference was nearly the same at $20 \mathrm{~mm} / \mathrm{min}$, with

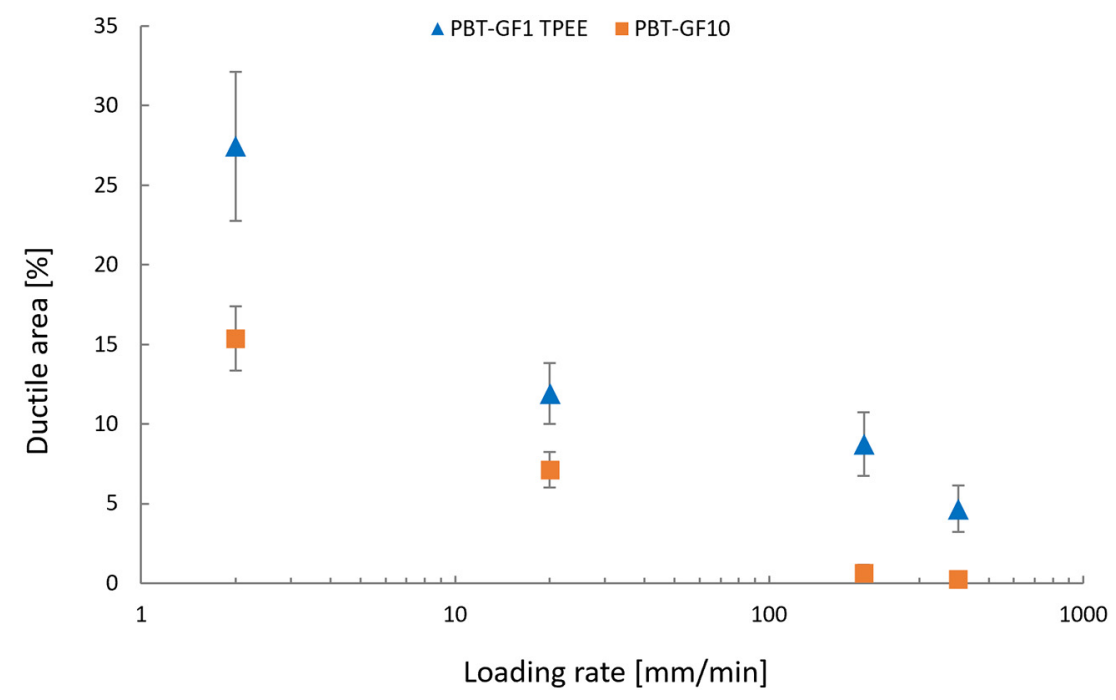

Fig. 11. Effect of loading rate on size of ductile areas. 
the size of ductile zone decreased by 56\% and 54\% for PBT-GF10 TPEE and PBT-GF10, respectively. At higher loading rates - 200 and $400 \mathrm{~mm} / \mathrm{min}$ - the ductile area of PBT-GF10 was nearly negligible, and much smaller than that in PBT-GF10 TPEE - by a factor of 14 and 18.8, respectively. In the latter composite, these ductile areas still occupied a noticeable part of the failed cross-sections - $10 \%$ at $200 \mathrm{~mm} / \mathrm{min}$ and $5 \%$ at $400 \mathrm{~mm} / \mathrm{min}$.

A rubbery material in a polymeric composite acts as stress concentrator [28]. Micrographs showed that voids formation, observed in the ductile area, is higher for PBT/TPEE than for PBT-GF10. A post-failure deformation (strain softening) was observed for tests carried-out at low strain rates, whereas, at high strain rates, a clear brittle failure mode was observed with much shorter postdeformation behaviour for PBT/TPEE. This demonstrates that the cross-sectional load-bearing capability of the specimen TPEE was smaller than that of PBT GF10 due to higher deformation manifested in formation of much larger ductile areas. This behaviour was related to the material's viscous flow, with formation of voids (depending on the material's microstructure and chemical composition) and fibre-matrix debonding (weak interface) [13] [29].

It could be argued that the ductile areas for PBT/TPEE do not necessarily reflect the ductility of the material; on the contrary, they lead to premature fracture acting as large-scale defect in the matrix. However, with increasing strain rates, the ductile-area size decreased due to reduction in the material's response time to external stress, with diminished viscous flow. This can enhance the cross-sectional load-bearing capability of the specimen, leading to increased ductility in presence of rubber particles (TPEE) and weak interfacial fibre-matrix bonds, leading, in turn, to an increase in the strain-at-break value and an apparent increase in fracture toughness.

In contrast, the ductile areas in PBT-GF10 represent the ductile ability of the composite due to different viscoelastic behaviour in absence of the rubbery phase and better fibre-matrix adhesion, where a decrease in ductile area resulted in a decrease in elongation at break.

\section{Conclusions}

This paper focused on the rate-dependent tensile properties of two SGFR PBT composites: standard PBT-GF10 and one blended with 10\% TPEE. A detailed investigation covered quasi-static and dynamic behaviours of these composites; it vividly demonstrated and quantified the effect of loading rate and interfacial matrix-fibre bonding on the failure mechanism and behaviour.

Mechanical analysis at different loading rates showed that both composites were strain-rate sensitive. In general, there was a negative impact on tensile properties caused by blending TPEE with PBT-GF10 under quasi-static conditions. However, in dynamic loading, improvements of tensile properties were observed. The analysis showed that PBT-GF10 TPEE composite exhibited higher rate-sensitivity at loading rates between 2 and $200 \mathrm{~mm} / \mathrm{min}$, with a greater increase in the Young's modulus, strain at UTS and elongation at break.

Morphological analysis showed that the main failure mechanism of both composites was fibre-matrix debonding and matrix failure. There was no evidence of fibre failure on the micrographs of fracture surfaces. Interfacial adhesion was negatively affected by TPEE blending which led to lower values of the Young's modulus and deterioration of some other mechanical properties.

In addition to the difference in interfacial fibre-matrix adhesion, larger ductile areas were observed on the fracture surfaces of PBT-GF10 TPEE at all the studied loading rates compared to those in PBT-GF10. Although SEM analysis showed that the matrix of PBT-GF10 TPEE behaved in a more ductile manner, this material ductility was not translated into enhanced mechanical performance in tensile tests. Generally, ductile materials exhibit higher values of elongation at break, which was not found PBT-GF10 TPEE. Its matrix showed higher cavity deformation caused by fibre pull-outs, especially in ductile areas, proving that the greater size of ductile areas observed for this composite was mainly the cause of a higher content of voids, formed during plastic deformation.

The results indicate that formation of the ductile area is a time-dependent (loading-rate-dependent) process, in contrast to the onset of a brittle fracture, initiated in a very short time. This is confirmed by the decreasing size of ductile area with an increasing strain rate. When a stable crack growth dominates, the crack propagates much more rapidly through the specimen in a brittle manner, not allowing the matrix material to deform plastically and, therefore, leading to a final failure of the specimen. This can also explain the decrease in size of ductile areas and the enhancement in some tensile properties with increasing loading rate.

\section{References}

[1] L. Utracki, Commercial Polymer Blends ISBN 0412-81020 4, Chapman \& Hall, 1998 (doi:10.1002/(SICI)1097-0126(200004)49:4 < 417::AID PI355 > 3.0.CO;2-I).

[2] M. Xanthos, Interfacial agents for multiphase polymer systems: recent advances, Polym. Eng. Sci. 28 (1988) 1392-1400, https://doi.org/10.1002/pen. 760282108.

[3] S. Sethi, D.K. Rathore, B.C. Ray, Effects of temperature and loading speed on interface-dominated strength in fibre/polymer composites: an evaluation for in-situ environment, Mater. Des. 65 (2015) 617-626, https://doi.org/10.1016/j.matdes.2014.09.053.

[4] N.K. Kalfoglou, Thermomechanical studies of semicrystalline polyether-ester copolymers. Effect of thermal, mechanical, and solvent treatment, J. Appl. Polym. Sci. 21 (1977) 543-554, https://doi.org/10.1002/app.1977.070210220.

[5] J. Huang, J. Wang, Y. Qiu, D. Wu, Mechanical properties of thermoplastic polyester elastomer controlled by blending with poly(butylene terephthalate), Polym. Test. 55 (2016) 152-159, https://doi.org/10.1016/j.polymertesting.2016.08.020.

[6] G. Verma, B. Kulshreshtha, S. Tyagi, A.K. Ghosh, PBT/thermoplastic elastomer blends - mechanical, morphological, and rheological characterization, Polym.- 
Plast. Technol. Eng. 47 (2008) 969-977, https://doi.org/10.1080/03602550802274662.

[7] Z. Yu, X. Wang, D. Wu, Mechanical properties, impact fracture behavior, and morphology of long-polyimide-fiber-reinforced poly(butylene terephthalate) composites, J. Compos. Mater. 51 (2017) 3425-3439, https://doi.org/10.1177/0021998316687031.

[8] W. Ignaczak, K. Wï̈niewska, J. Janik, M. El Fray, Mechanical and thermal properties of PP/PBT blends compatibilized with triblock thermoplastic elastomer, Pol. J. Chem. Technol. 17 (2015) 78-83, https://doi.org/10.1515/pjct-2015-0053.

[9] Y. Bai, S. Lv, F. Liu, M.T. Run, Preparation, morphology and properties of poly(trimethylene terephthalate)/thermoplastic polyester elastomer blends, J. Macromol. Sci. B Phys. 53 (2014) 1553-1573, https://doi.org/10.1080/00222348.2014.939525.

[10] H. Shin, E.S. Park, Mechanical and dielectric breakdown properties of PBT/TPE, PBT/PBT/PET, and PBT/antioxidant blends, J. Appl. Polym. Sci. 114 (2009) 3008-3015, https://doi.org/10.1002/app.30930.

[11] N.M. Barkoula, B. Alcock, N.O. Cabrera, T. Peijs, Morphology, mechanical, and dielectric breakdown properties of PBT/PET/TPE, PBT/PET/PA66, PBT/PET/ LMPE, and PBT/PET/TiO2 blends, Polym. Polym. Compos. 16 (2008) 101-113, https://doi.org/10.1002/pc.

[12] J. Fitoussi, M. Bocquet, F. Meraghni, Effect of the matrix behavior on the damage of ethylene-propylene glass fiber reinforced composite subjected to high strain rate tension, Compos. B Eng. 45 (2013) 1181-1191, https://doi.org/10.1016/j.compositesb.2012.06.011.

[13] M. Schoßig, C. Bierögel, W. Grellmann, T. Mecklenburg, Mechanical behavior of glass-fiber reinforced thermoplastic materials under high strain rates, Polym. Test. 27 (2008) 893-900, https://doi.org/10.1016/j.polymertesting.2008.07.006.

[14] J.L. Thomason, The influence of fibre length, diameter and concentration on the impact performance of long glass-fibre reinforced polyamide 6,6, Compos. A Appl. Sci. Manuf. 40 (2009) 114-124, https://doi.org/10.1016/j.compositesa.2008.10.013.

[15] J.J. Horst, J.L. Spoormaker, Mechanisms of fatigue in short glass fiber reinforced polyamide 6, Polym. Eng. Sci. 36 (1996) 2718-2726, https://doi.org/10.1002/ pen.10671.

[16] N. Sato, T. Kurauchi, S. Sato, O. Kamigaito, Microfailure behaviour of randomly dispersed short fibre reinforced thermoplastic composites obtained by direct SEM observation, J. Mater. Sci. 26 (1991) 3891-3898.

[17] N. Sato, T. Kurauchi, S. Sato, O. Kamigaito, Mechanism of fracture of short glass fibre-reinforced polyamide thermoplastic, J. Mater. Sci. 19 (1984) 1145-1152, https://doi.org/10.1007/BF01120023.

[18] ISO-527-1, Plastics-Determination of tensile properties-Part 1: General principles, (1997).

[19] H. Rolland, N. Saintier, G. Robert, Damage mechanisms in short glass fibre reinforced thermoplastic during in situ microtomography tensile tests, Compos. B Eng. 90 (2016) 365-377, https://doi.org/10.1016/j.compositesb.2015.12.021.

[20] J.E. Yetman, A.J. Sobey, J.I.R. Blake, R.A. Shenoi, Mechanical and fracture properties of glass vinylester interfaces, Compos. B Eng. 130 (2017) 38-45, https:// doi.org/10.1016/j.compositesb.2017.07.011.

[21] J.L. Thomason, The influence of fibre length, diameter and concentration on the modulus of glass fibre reinforced polyamide 6,6, Compos. A Appl. Sci. Manuf. 39 (2008) 1732-1738, https://doi.org/10.1016/j.compositesa.2008.08.001.

[22] C. Chen, C. Zhang, C. Liu, Y. Miao, S.C. Wong, Y. Li, Rate-dependent tensile failure behavior of short fiber reinforced PEEK, Compos. B Eng. 136 (2018) 187-196, https://doi.org/10.1016/j.compositesb.2017.10.031.

[23] J.T. Fan, J. Weerheijm, L.J. Sluys, Glass interface effect on high-strain-rate tensile response of a soft polyurethane elastomeric polymer material, Compos. Sci. Technol. 118 (2015) 55-62, https://doi.org/10.1016/j.compscitech.2015.08.007.

[24] P. Guoliang, G. Qiang, T. Aiguo, H. Zhiqiang, Mechanical behaviors of Al2O3nanoparticles reinforced polyetheretherketone, Mater. Sci. Eng. A 492 (2008) 383-391, https://doi.org/10.1016/j.msea.2008.05.026.

[25] Z. Wang, J. Yao, N. Tian, J. Zheng, P. Gao, Mechanical behavior and damage evolution for granite subjected to cyclic loading, Adv. Mater. Sci. Eng. 2018 (2018) 1-10, https://doi.org/10.1155/2018/4312494.

[26] A. Schaaf, M. De Monte, C. Hoffmann, M. Vormwald, M. Quaresimin, Damage mechanisms in PBT-GF30 under thermo-mechanical cyclic loading, AIP Conf. Proc, 2014, pp. 600-605, , https://doi.org/10.1063/1.4873852.

[27] J.J. Horst, J.L. Spoormaker, Fatigue fracture mechanisms and fractography of short-glassfibre-reinforced polyamide 6, J. Mater. Sci. 32 (1997) 3641-3651, https://doi.org/10.1023/a:1018634530869.

[28] J. Yang, J. Liu, Cavitation of rubber particles in high-impact polystyrene, Polym. J. 33 (2001) 952-954, https://doi.org/10.1295/polymj.33.952.

[29] A. Pawlak, Plastic deformation and cavitation in semicrystalline polymers studied by X-ray methods, Polimery/Polymers 59 (2014) 533-541, https://doi.org/10. 14314/polimery.2014.533. 